\title{
Exponential Dichotomy and Eberlein-Weak Almost Periodic Solutions*
}

\author{
Elhadi Ait Dads ${ }^{1}$, Samir Fatajou ${ }^{2}$, Lahcen Lhachimi ${ }^{1}$ \\ ${ }^{1}$ Départment de Mathématiques, Faculté des Sciences Semlalia, Université Cadi Ayyad, Marrakesh, Morocco \\ ${ }^{2}$ Laboratoire L. M. C., Départment de Mathématiques et Informatique, Faculté Polydisciplinaire-Safi (FPS) \\ Université Cadi Ayyad, Safi, Morocco \\ Email: aitdads@uca.ma,s.fatajou@ucam.ac.ma, 1llahcen@gmail.com
}

Received July 26, 2012; revised August 26, 2012; accepted September 4, 2012

\section{ABSTRACT}

We give sufficient conditions ensuring the existence and uniqueness of an Eberlein-weakly almost periodic solution to the following linear equation

$$
\frac{\mathrm{d} x}{\mathrm{~d} t}(t)=A(t) x(t)+f(t),
$$

in a Banach space $X$, where $(A(t))_{t \in \mathbb{R}}$ is a family of infinitesimal generators such that for all $t \in \mathbb{R}, A(t+T)=A(t)$ for some $T>0$, for which the homogeneuous linear equation $\frac{\mathrm{d} x}{\mathrm{~d} t}(t)=A(t) x(t)$ is well posed, stable and has an exponential dichotomy, and $f: \mathbb{R} \rightarrow X$ is Eberlein-weakly amost periodic.

Keywords: Bounded Solutions; Almost Periodic and Eberlein Weak Almost Periodic Functions; Exponential Dichotomy; Linear Differential Equations

\section{Introduction}

The aim of this work is to investigate the existence and uniqueness of a weakly almost periodic solution in the sense of Eberlein for the following linear equation :

$$
\frac{\mathrm{d} x}{\mathrm{~d} t}(t)=A(t) x(t)+f(t), \text { for all } t \in \mathbb{R}
$$

for $x \in X$, where $X$ is a complex Banach space, $A(t)$ is (unbounded) linear operator acting on $X$ for every fixed $t \in \mathbb{R}$ such that for all $t \in \mathbb{R}, A(t+T)=A(t)$ for some $T>0$, and the input function $f: \mathbb{R} \rightarrow X$ is weakly almost periodic in the sense of Eberlein (Eberlein-weakly almost periodic). In the sequel, we essentially assume that:

$\left(H_{1}\right) \quad(A(t))_{t \in \mathbb{R}}$ is a family of infinitesimal generators for which the corresponding homogeneous equation of (1) is well posed and stable in the following sense: there exists a $T$-periodic strongly continuous evolutionary process $(U(t, s))_{t \geq s}$, which is uniformly bounded and strongly continuous such in particular that:

\footnotetext{
${ }^{*}$ The authors were partially supported by the IRD project UMMISCO, UMI 209.
}

for all

$$
t \geq s, U(t, t)=I_{X}, U(t+T, s+T)=U(t, s)
$$

and

$$
U(t, s) U(s, \tau)=U(t, \tau) \text {, for all } t \geq s \geq \tau .
$$

Further, if $x \in D(A(s))$ is given and $t \geq s$, then $U(t, s) x \in D(A(t))$, and

$$
\left\{\begin{array}{l}
\frac{\partial}{\partial t} U(t, s) x=A(t) U(t, s) x, \\
\frac{\partial}{\partial s} U(t, s) x=-U(t, s) A(s) x .
\end{array}\right.
$$

We also assume

$\left(H_{2}\right)$ The corresponding homogeneous equation of (1) has an exponential dichotomy, i.e., there exist a family of projections $Q(t), \quad t \in \mathbb{R}$ and positive constants $M, \alpha$ such that the following conditions are satisfied :

1) For every fixed $x \in X$ the map $t \mapsto Q(t) x$, is continuous and $T$-periodic,

2) $Q(t) U(t, s)=U(t, s) Q(s)$,

$$
\forall(t, s) \in \Delta:=\left\{(t, s) \in \mathbb{R}^{2}: t \geq s\right\},
$$

3) $\|U(t, s) P(s)\| \leq M e^{-\alpha(t-s)}, \forall(t, s) \in \Delta$, where for all 
$t \in \mathbb{R}$ and $x \in X, P(t) x:=x-Q(t) x$,

4) $\|U(t, s) Q(s)\| \leq M e^{\alpha(t-s)}, \forall(s, t) \in \Delta$,

5) $\left.U(t, s)\right|_{\operatorname{Im} Q(s)}$ is an isomorphism from $\operatorname{Im} Q(s)$ onto $\operatorname{Im} Q(t), \forall(s, t) \in \Delta$.

The problem of the existence of almost periodic solutions has been extensively studies in the literature [1-6]. Eberlein-weak almost periodic functions are more general than almost periodic functions and they were introduced by Eberlein [7], for more details about this topics we refer to [8-11] where the authors gave an important overview about the theory of Eberlein weak almost periodic functions and their applications to differential equations. In the literature, many works are devoted to the existence of almost periodic and pseudo almost periodic solutions for differential equations (a pseudo almost periodic function is the sum of an almost periodic function and of an ergodic perturbation), but results about Eberlein weak almost periodic solutions are rare [7,12-16].

In ([17], Chap. 3) the authors investigate the existence and uniqueness of an almost periodic solution for equation (1) when the corresponding homogeneous equation of (1) has an exponential dichotomy and the function $f$ is almost periodic. In ([17], Chap. 3) the authors showed that, if the corresponding homogeneous equation of (1) has an exponential dichotomy and the function $f$ is almost periodic, the equation (1) has a unique bounded integral solution on $\mathbb{R}$ which is also almost periodic. Here we propose to extend the result in [17] to the Eberleinweakly almost periodic case.

\section{Eberlein-Weak Almost Periodic Functions}

In the sequel, we give some properties about weak almost periodic functions in the sense of Eberlein (Eberlein-weak almost periodic functions).

Let $X$ and $Y$ be two Banach spaces. Denote by $C(X, Y)$ the space of all continuous functions from $X$ to $Y$. Let $B C(\mathbb{R}, X)$ be the space of all bounded and continuous functions from $\mathbb{R}$ to $X$, equipped with the norm of uniform topology.

Definition 2.1 A bounded continuous function $x: \mathbb{R} \rightarrow X$ is said to be almost periodic, if the orbit of $x$, the set of translates of $x$ :

$$
O(x):=\left\{x_{\tau}:=\{t \rightarrow x(t+\tau)\}: \tau \in \mathbb{R}\right\}
$$

is a relatively compact set in $B C(\mathbb{R}, X)$ with respect to the supremum norm.

We denote these functions by

$$
A P(\mathbb{R}, X):=\{x \in B C(\mathbb{R}, X): x \text { is almost periodic }\}
$$

Definition 2.2 A function $x \in B C(\boldsymbol{J}, X)$, for
$\boldsymbol{J} \in\left\{\mathbb{R}, \mathbb{R}^{+}\right\}$is said to be weakly almost periodic in the sense of Eberlein (Eberlein-weakly almost periodic) if the orbit of $x$ with respect to $\mathbf{J}$ :

$$
O_{J}(x):=\left\{x_{\tau}:=\{t \rightarrow x(t+\tau)\}: \tau \in \boldsymbol{J}\right\}
$$

is relatively compact with respect to the weak topology of the sup-normed Banach space $B C(J, X)$.

For the sequel, $W(\boldsymbol{J}, X)$ will denote the set of Eberlein-weakly almost periodic $X$ valued functions.

Theorem 2.3 Equipped with the norm

$$
\|x\|_{\infty}:=\sup _{t \in \mathbb{R}}\|x(t)\|,
$$

the vector space $W(\boldsymbol{J}, X)$ is a Banach space.

In $[18,19]$ Deleeuw and Glicksberg proved that if we consider the subspace of those Eberlein weakly almost periodic functions, which contain zero in the weak closure of the orbit (weak topology of $\left(B C(\boldsymbol{J}, X),\|\cdot\|_{\infty}\right)$ ), i.e.;

$$
\begin{aligned}
& W_{0}(\boldsymbol{J}, X) \\
& :=\left\{x \in W(\boldsymbol{J}, X): \text { for a sequence }\left(s_{n}\right)_{n \in \mathbb{R}} \subseteq \boldsymbol{J}, x_{s_{n}} \rightarrow 0\right\},
\end{aligned}
$$

the following decomposition

$$
W(\boldsymbol{J}, X)=\left.A P(\mathbb{R}, X)\right|_{J}+W_{0}(\boldsymbol{J}, X)
$$

holds. Moreover, if $x \in W(\boldsymbol{J}, X), x^{a} \in A P(\boldsymbol{J}, X)$, and $x_{0} \in W_{0}(J, X)$ with $x=\left.x^{a}\right|_{J}+x_{0}$, then

$$
\|\cdot\|-\lim _{T \rightarrow \infty} \frac{1}{T} \int_{0}^{T}\left(x_{t}(s)-x_{0}(s)\right) \mathrm{d} s=0,
$$

uniformly in $t \in \boldsymbol{J}$.

For a more detailed information about the decomposition and the ergodic result we refer to the book of Krengel [20,21].

In order to prove the weak compactness of the translates, Ruess and Summers extended the double limits criterion of Grothendieck [22] to the following:

Proposition 2.4 A subset $H \subset B C(\boldsymbol{J}, X)$, is relatively weakly compact if and only if,

1) $H$ is bounded in $B C(\boldsymbol{J}, X)$, and

2) for all $\left(h_{m}\right)_{m \in \mathbb{N}} \subseteq H, \quad\left(t_{n}\right)_{n \in \mathbb{N}} \subseteq \boldsymbol{J}$, and $\left(x_{n}^{*}\right)_{n \in \mathbb{N}} \subseteq \boldsymbol{B}_{X^{*}}$, the following double limits condition holds:

$$
\lim _{m \rightarrow \infty} \lim _{n \rightarrow \infty}\left\langle h_{m}\left(t_{n}\right), x_{n}^{*}\right\rangle=\lim _{n \rightarrow \infty} \lim _{m \rightarrow \infty}\left\langle h_{m}\left(t_{n}\right), x_{n}^{*}\right\rangle,
$$

whenever the iterated limits exist.

This result will be the main tool in verifying weak almost periodicity. For the other task we will use.

Proposition 2.5 For every Eberlein weakly almost periodic function $f$ there exists a sequence $\left(t_{n}\right)_{n \in \mathbb{N}}$ such that if $g$ is the almost periodic part $f_{t_{n}} \rightarrow g$. 


\section{Statement of the Main Result}

In this section, we state a result of the existence and uniqueness of an Eberlein-weakly almost periodic solution of the Periodic Inhomogeneous Linear Equation (1). The existence and uniqueness of an almost periodic and bounded solution has been studied by M. N'Guérékata ([17]). More precisely, the author proved the following result.

Theorem 3.1 ([17]) Assume that $\left(H_{1}\right)$ and $\left(H_{2}\right)$ hold. If the function $f$ is continuous and bounded on $\mathbb{R}$, then Equation (1) has a unique bounded mild solution $x_{f}$ on $\mathbb{R}$. Moreover, if $f$ is almost periodic, then $x_{f}$ is almost periodic.

We propose to extend the above theorem to the case where $f$ is Eberlein-weakly almost periodic.

Theorem 3.2 Assume that $\left(H_{1}\right)$ and $\left(H_{2}\right)$ hold. If the function $f$ is Eberlein-weakly almost periodic with a relatively compact range, then Equation (1) has a unique bounded mild solution $x_{f}$ on $\mathbb{R}$ which is Eberleinweakly almost periodic.

For the proof of theorem (3.2), we use the following lemmas.

Lemma 3.3 Let $f: \boldsymbol{J} \rightarrow X$ be a bounded uniformly continuous function with relatively compact range, $\left(t_{m}\right)_{m \in \mathbb{N}} \subseteq \boldsymbol{J},\left(w_{n}\right)_{n \in \mathbb{N}} \subseteq \boldsymbol{J}$, and $\left(x_{m}^{*}\right)_{m \in \mathbb{N}} \subseteq \boldsymbol{B}_{X^{*}}$. If $\left(t_{m}\right)_{m \in \mathbb{N}}$, or $\left(w_{n}\right)_{n \in \mathbb{N}}$ is bounded, then one has

$$
\lim _{m \rightarrow \infty} \lim _{n \rightarrow \infty}\left\langle f\left(t_{m}+w_{n}\right), x_{m}^{*}\right\rangle=\lim _{n \rightarrow \infty} \lim _{m \rightarrow \infty}\left\langle f\left(t_{m}+w_{n}\right), x_{m}^{*}\right\rangle,
$$

whenever the iterated limits exist.

Proof. Noting that only the equality of the iterated limits has to be proved, we may pass to subsequences. Therefore we assume that the following limits exists

1) $\omega-\lim _{n \rightarrow \infty} \omega-\lim _{m \rightarrow \infty} f\left(t_{m}+w_{n}\right):=a$,

2) $\omega-\lim _{m \rightarrow \infty} \omega-\lim _{n \rightarrow \infty} f\left(t_{m}+w_{n}\right):=b$,

3) $\omega-\lim _{m \rightarrow \infty} x_{m}^{*}=x^{*}$ and

4) $\lim _{m \rightarrow \infty} t_{m}=t$, or $\lim _{n \rightarrow \infty} w_{n}=w$.

here 1) and 2) can be obtained by a diagonalization argument. Since $f(\boldsymbol{J})$ is separable, we may assume that 3) holds.

Let $\lim _{m \rightarrow \infty} t_{m}=t$; then by the uniform continuity of $f$, we find

$$
\lim _{m \rightarrow \infty}\left\|f_{t_{m}}-f_{t}\right\|_{\infty}=0
$$

and

$$
\lim _{m \rightarrow \infty} \lim _{n \rightarrow \infty}\left\langle f\left(t_{m}+w_{n}\right), x_{m}^{*}\right\rangle=\lim _{n \rightarrow \infty} \lim _{m \rightarrow \infty}\left\langle f\left(t_{m}+w_{n}\right), x_{m}^{*}\right\rangle .
$$

Again by uniform continuity of $f$, and by the choice of subsequences we find for the interchanged limits.

Lemma 3.4 Let $\left(f_{n}\right)_{n \in \mathbb{N}} \subset B C(\boldsymbol{J}, X)$ such that for a subcompact set $\mathbf{K} \subset X$

$$
\bigcup_{t \in J, n \in \mathbb{N}}\left\{f_{n}(t)\right\} \subset \boldsymbol{K},
$$

and

$$
f_{n} \rightarrow 0 \text { in } B C(\boldsymbol{J}, X) .
$$

Then $\left\|f_{n}\right\| \rightarrow 0$ in $B C(\boldsymbol{J})$.

Proof. We first prove that the set $\left(\left\|f_{n}(.)\right\|\right)_{n \in \mathbb{N}}$ is weakly relatively compact in $B C(\boldsymbol{J})$. Thus, for given sequences $\left(n_{j}, t_{m}\right)_{j, m \in \mathbb{N}} \subseteq \mathbb{N} \times \boldsymbol{J}$, we have to verify the following identity :

$$
\lim _{m \rightarrow \infty} \lim _{j \rightarrow \infty}\left\|f_{n_{j}}\left(t_{m}\right)\right\|=\lim _{j \rightarrow \infty} \lim _{m \rightarrow \infty}\left\|f_{n_{j}}\left(t_{m}\right)\right\|,
$$

whenever the iterated limits exist. Since

$x_{j, m}:=f_{n_{j}}\left(t_{m}\right) \in \boldsymbol{K}$ for all $j, m \in \mathbb{N}$, as a consequence of the metric weak compactness of $\boldsymbol{K}$, we may pass to subsequences of $\left(n_{j}\right)_{j \in \mathbb{N}}$ and $\left(t_{m}\right)_{m \in \mathbb{N}}$ such that the iterated limits of $\left(x_{j, m}\right)_{j, m \in \mathbb{N}}$ exist in $X$, without loss of generality the sequences are chosen in this way. The characterization of weak compactness gives,

$$
\lim _{m \rightarrow \infty} \lim _{j \rightarrow \infty} x_{j, m}=\lim _{j \rightarrow \infty} \lim _{m \rightarrow \infty} x_{j, m} .
$$

Since $(\boldsymbol{K}$, week $)=(\boldsymbol{K},\|\|$.$) \quad (the convergence holds in$ norm), hence one will obtain that $\left(\left\|f_{n}(.)\right\|\right)_{n \in \mathbb{N}}$ is weakly relatively compact in $B C(\boldsymbol{J})$. Using the fact that :

$$
\lim _{n \rightarrow \infty}\left\|f_{n}(x)\right\|=0,
$$

a standard trick of topology gives $\left\|f_{n}().\right\| \rightarrow$ in $B C(\boldsymbol{J})$.

Lemma 3.5 Let, for a Banach space $X, Y=L(X)$, (the space of all bounded linear operators acting on $X$ ) and $A(.) \in B C(\mathbb{R}, Y) T$ - periodic. Then, for any given $g: \mathbb{R} \rightarrow X$ Eberlein-weakly almost periodic with a relatively compact range,

$$
\{t \rightarrow A(t) g(t)\} \in W(\mathbb{R}, X) .
$$

Proof. In Order to prove that $\{t \rightarrow A(t) g(t)\}$ is Eberlein-weakly almost periodic, by W. M. Ruess and W. H. Summers's criterion (2.4), we have to verify that for given sequences $\left(t_{m}\right)_{m \in \mathbb{N}} \subseteq \mathbb{R}, \quad\left(w_{n}\right)_{n \in \mathbb{N}} \subseteq \mathbb{R}$, and $\left(x_{n}^{*}\right)_{n \in \mathbb{N}} \subseteq \boldsymbol{B}_{X^{*}}$

$$
\begin{aligned}
& \lim _{m \rightarrow \infty} \lim _{n \rightarrow \infty}\left\langle A\left(t_{m}+w_{n}\right) g\left(t_{m}+w_{n}\right), x_{n}^{*}\right\rangle \\
& =\lim _{n \rightarrow \infty} \lim _{m \rightarrow \infty}\left\langle A\left(t_{m}+w_{n}\right) g\left(t_{m}+w_{n}\right), x_{n}^{*}\right\rangle,
\end{aligned}
$$


whenever the iterated limits exist. Assuming that the iterated limits exist and by the fact that we only have to prove the equality of them, we may pass to subsequences for the verification. Since $g$ is Eberlein weakly almost periodic with a relatively compact range, by a use of a diagonalization routine, we may assume that

$$
\lim _{n \rightarrow+\infty} \lim _{m \rightarrow+\infty} g\left(t_{k_{m}}+w_{l_{n}}\right)=\lim _{m \rightarrow+\infty} \lim _{n \rightarrow+\infty} g\left(t_{k_{m}}+w_{l_{n}}\right)=z,
$$

for a suitable choice of subsequences $\left\{t_{k_{m}}\right\}_{m \in \mathbb{N}}$ and $\left\{t_{n}\right\}_{n \in \mathbb{N}}$. We define

$$
\left\{\begin{array}{l}
b_{n, m}:=\left\langle A\left(t_{k_{m}}+w_{l_{n}}\right) g\left(t_{k_{m}}+w_{l_{n}}\right), x_{m}^{*}\right\rangle, \\
b=\lim _{n \rightarrow+\infty} \lim _{m \rightarrow+\infty} b_{n, m}, \\
a_{n, m}:=\left\langle A\left(t_{k_{m}}+w_{l_{n}}\right) z, x_{m}^{*}\right\rangle .
\end{array}\right.
$$

By hypothesis, we have $t \rightarrow A(t) z$ is periodic, thus $\left\{a_{n, m}\right\}_{n, m \in \mathbb{N}}$ satisfies the double limits condition. Let $a \in \mathbb{R}$ be the double limit.

Now,

$$
|b-a| \leq\left|b-b_{n, m}\right|+\left|a-a_{n, m}\right|+\left|a_{n, m}-b_{n, m}\right| .
$$

From the convergence of $\left\{a_{n, m}\right\}_{n, m \in \mathbb{N}}$ and $\left\{b_{n, m}\right\}_{n, m \in \mathbb{N}}$, we derive that for every $\varepsilon>0$ there exists an $n_{0} \in \mathbb{N}$, such that for $n \geq n_{0}$, there exist an $m_{n}$, such that

$$
\left\{\begin{array}{c}
\left|b_{n, m}-b\right| \leq \frac{\varepsilon}{3} \forall m \geq m_{n}, \\
\text { and } \\
\left|a_{n, m}-a\right| \leq \frac{\varepsilon}{3} \forall m \geq m_{n} .
\end{array}\right.
$$

Using the double limits condition of the sequence $\left\{x_{n, m}\right\}_{n, m \in \mathbb{N}}$, for given $\delta>0$, there exists $n_{1} \in \mathbb{N}$, such that for all $n \geq n_{1}$, there is an $m_{n}^{1}$, such that $\left\|x_{n, m}-z\right\|<\delta$, for all $m \geq m_{n}^{1}$. Applying the continuity of the map $x \in \overline{g(\mathbb{R})}^{\omega} \mapsto\{t \rightarrow A(t) x\}$, for $\varepsilon>0$, we find a $\delta>0$, and according to the previous observation, there exists an $n_{1} \in \mathbb{N}$, such that for all $n \geq n_{1}$, we find an $m_{n}^{1}$, with

$$
\sup _{t \in \mathbb{R}}\left\|A(t) x_{n, m}-A(t) z\right\|<\varepsilon \text {, for every } m \geq m_{n}^{1} .
$$

This yields, by a standard estimate, that $|b-a|<\varepsilon$, and hence $b=a$.

The following example shows that the compactness assumption on the range of $g$ is essential and that the periodicity of $\{A(t)\}_{t \in \mathbb{R}}$ is not sufficient even if additional algebraic structure is given.

Example 3.6 We let $X=£^{2}$ and choose

$$
\begin{aligned}
A(t)\left(\left\{x_{n}\right\}_{n \in \mathbb{N}}\right): & =\left\{\exp (\text { int })\left\{x_{n}\right\}\right\}_{n \in \mathbb{N}}, \text { for all } t \in \mathbb{N}, \\
& \text { and }\left\{x_{n}\right\}_{n \in \mathbb{N}} \in \mathfrak{£}^{2} .
\end{aligned}
$$

Further, if $\chi_{A}$ denotes the indicator function for the set $A$, we choose $g \in B C(\mathbb{R}, X)$

$$
g(t):=\left\{\sin ^{2}(t) \chi_{[n \pi,(n+1) \pi))}\right\}_{n \in \mathbb{N}} .
$$

Using Lemma 2. 16 in ([13]), we obtain that $g$ is Eberlein weakly almost periodic. Now, for the sequences

$$
s_{n}:=\left(n+\frac{1}{2}\right) \pi+\frac{1}{n}, t_{n}:=\left(n+\frac{1}{2}\right) \pi-\frac{1}{n},
$$

some calculations lead to the identity :

$$
\lim _{n \rightarrow \infty}\left\|A\left(s_{n}\right) g\left(s_{n}\right)-A\left(t_{n}\right) g\left(t_{n}\right)\right\|=\frac{2}{\sin (1)},
$$

hence $t \rightarrow A(t) g(t)$ is not uniformly continuous, hence not Eberlein weakly almost periodic.

Proof. (of Theorem 3.2) Since $f$ is Eberlein-weakly almost periodic, then $f$ is continuous and bounded on $\mathbb{R}$. The existence and uniqueness of the bounded mild solution $x_{f}$ on $\mathbb{R}$ result of theorem (3.1).

We claim that

$\forall t \in \mathbb{R}$

$$
x_{f}(t)=\int_{-\infty}^{t} U(t, s) P(s) f(s) \mathrm{d} s-\int_{t}^{+\infty} U(t, s) Q(s) f(s) \mathrm{d} s .
$$

In fact, for any $t \geq s$ we have

$$
\begin{aligned}
& x_{f}(t)=\int_{-\infty}^{t} U(t, \tau) P(\tau) f(\tau) \mathrm{d} \tau \\
& -\int_{t}^{+\infty} U(t, \tau) Q(\tau) f(\tau) \mathrm{d} \tau \\
& =U(t, s)\left(\int_{-\infty}^{s} U(s, \tau) P(\tau) f(\tau) \mathrm{d} \tau\right. \\
& \left.-\int_{s}^{-\infty} U(s, \tau) Q(\tau) f(\tau) \mathrm{d} \tau\right) \\
& \left.+\int_{s}^{t} U(t, \tau) P(\tau) f(\tau) d \tau+\int_{s}^{t} U(t, \tau) Q(\tau) f(\tau) \mathrm{d} \tau\right) \\
& =U(t, s) x_{f}(s)+\int_{s}^{t} U(t, \tau) f(\tau) \mathrm{d} \tau .
\end{aligned}
$$

On the other hand, we have

$$
\begin{aligned}
\left\|x_{f}(t)\right\| & \leq M \int_{-\infty}^{+\infty} \mathrm{e}^{-\alpha|t-s|}\|f(s)\| \mathrm{d} s \\
& \leq M \int_{-\infty}^{+\infty} \mathrm{e}^{-\alpha|\zeta|}\|f(t-\zeta)\| \mathrm{d} \zeta .
\end{aligned}
$$


which ends the claim.

Now, to complete the proof, it remains for us to prove that $x_{f}$ is Eberlein-weakly almost periodic. By Ruess and Summers's double limits criterion, we have to verify that for given sequences

$$
\begin{gathered}
\left(w_{n}\right)_{n \in \mathbb{N}} \subseteq \mathbb{R},\left(t_{m}\right)_{m \in \mathbb{N}} \subseteq \mathbb{R} \text { and }\left(x_{m}^{*}\right)_{m \in \mathbb{N}} \subseteq \boldsymbol{B}_{X^{*}}, \\
\lim _{m \rightarrow \infty} \lim _{n \rightarrow \infty}\left\langle x_{f}\left(w_{n}+t_{m}\right), x_{m}^{*}\right\rangle \\
=\lim _{n \rightarrow \infty} \lim _{m \rightarrow \infty}\left\langle x_{f}\left(w_{n}+t_{m}\right), x_{m}^{*}\right\rangle
\end{gathered}
$$

$$
\begin{aligned}
& \left\|x_{f}\left(t_{m}+k_{n} T+r\right)-x_{f}\left(t_{m}+k_{l} T+r\right)\right\| \\
& \leq\left\|\iint_{-\infty}^{t_{m}+k_{n} T+r} U\left(t_{m}+k_{n} T+r, s\right) P(s) f(s) \mathrm{d} s-\int_{-\infty}^{t_{m}+k_{l} T+r} U\left(t_{m}+k_{l} T+r, s\right) P(s) f(s) \mathrm{d} s\right\| \\
& \leq\left\|\iint_{-\infty}^{t_{m}+k_{n} T+r} U\left(t_{m}+k_{n} T+r, s\right) P(s) f(s) \mathrm{d} s-\int_{-\infty}^{t_{m}+k_{l} T+r} U\left(t_{m}+k_{l} T+r, s\right) P(s) f(s) \mathrm{d} s\right\| \\
& \quad+\left\|\int_{t_{m}+k_{n} T+r}^{+\infty} U\left(t_{m}+k_{n} T+r, s\right) Q(s) f(s) \mathrm{d} s-\int_{t_{m}+k_{l} T+r}^{+\infty} U\left(t_{m}+k_{l} T+r, s\right) Q(s) f(s) \mathrm{d}\right\| \| \\
& \quad+\left\|\int_{t_{m}+k_{n} T+r}^{+\infty} U\left(t_{m}+k_{n} T+r, s\right) Q(s) f(s) \mathrm{d} s-\int_{t_{m}+k_{l} T+r}^{+\infty} U\left(t_{m}+k_{l} T+r, s\right) Q(s) f(s) \mathrm{d}\right\| \|
\end{aligned}
$$

Since $\forall t \geq s, \quad U(t+T, s+T)=U(t, s)$, we obtain:

$$
\begin{aligned}
& \left\|x_{f}\left(t_{m}+k_{n} T+r\right)-x_{f}\left(t_{m}+k_{l} T+r\right)\right\| \\
& \leq\left\|\int_{-\infty}^{t_{m}} U\left(t_{m}+r, \tau+r\right)\left(P\left(\tau+k_{n} T+r\right) f\left(\tau+k_{n} T+r\right)-P\left(\tau+k_{l} T+r\right) f\left(\tau+k_{l} T+r\right)\right) \mathrm{d} \tau\right\| \\
& \leq\left\|\int_{-\infty}^{t_{m}} U\left(t_{m}+r, \tau+r\right)\left(P\left(\tau+k_{n} T+r\right) f\left(\tau+k_{n} T+r\right)-P\left(\tau+k_{l} T+r\right) f\left(\tau+k_{l} T+r\right)\right) \mathrm{d} \tau\right\| \\
& \quad+\left\|\int_{t_{m}}^{+\infty} U\left(t_{m}+r, \tau+r\right)\left(P\left(\tau+k_{n} T+r\right) f\left(\tau+k_{n} T+r\right)-P\left(\tau+k_{n} T+r\right) f\left(\tau+k_{n} T+r\right)\right) \mathrm{d} \tau\right\| \\
& \quad+\left\|\int_{t_{m}}^{+\infty} U\left(t_{m}+r, \tau+r\right)\left(P\left(\tau+k_{n} T+r\right) f\left(\tau+k_{n} T+r\right)-P\left(\tau+k_{n} T+r\right) f\left(\tau+k_{n} T+r\right)\right) \mathrm{d} \tau\right\|
\end{aligned}
$$

thus,

$$
\left\|x_{f}\left(t_{m}+k_{n} T+r\right)-x_{f}\left(t_{m}+k_{l} T+r\right)\right\| \leq M\left(I_{1}+I_{2}\right) .
$$

where

$$
I_{1}=\int_{-\infty}^{t_{m}} e^{-\alpha\left(t_{m}-\tau\right)}\left\|P\left(\tau+k_{n} T+r\right) f\left(\tau+k_{n} T+r\right)-P\left(\tau+k_{l} T+r\right) f\left(\tau+k_{l} T+r\right)\right\| \mathrm{d} \tau
$$

and

$$
I_{2}=\int_{t_{m}}^{+\infty} e^{\alpha\left(t_{m}-\tau\right)}\left\|Q\left(\tau+k_{n} T+r\right) f\left(\tau+k_{n} T+r\right)-Q\left(\tau+k_{n} T+r\right) f\left(\tau+k_{n} T+r\right)\right\| \mathrm{d} \tau
$$

Since by Lemma (3.5)

$$
\{t \rightarrow P(t) f(t)\}
$$

and

$$
\{t \rightarrow Q(t) f(t)\}
$$

are Eberlein weakly almost periodic, we may assume that

$$
P\left(k_{n} T+.\right) f\left(k_{n} T+.\right) \rightarrow g_{P}
$$

and

$$
Q\left(k_{n} T+.\right) f\left(k_{n} T+.\right) \rightarrow g_{Q}
$$

Bringing the last estimate into play we obtain 


$$
\begin{aligned}
& \left\|x_{f}\left(t_{m}+k_{n} T+r\right)-x_{f}\left(t_{m}+k_{l} T+r\right)\right\| \\
& \leq \int_{t_{-\infty}}^{t_{m}} e^{-\alpha\left(t_{m}-\tau\right)}\left\|P\left(\tau+k_{n} T+r\right) f\left(\tau+k_{n} T+r\right)-g_{P}(\tau)\right\| \mathrm{d} \tau \\
& \leq \int_{t_{-\infty}}^{t_{m}} e^{-\alpha\left(t_{m}-\tau\right)}\left\|P\left(\tau+k_{n} T+r\right) f\left(\tau+k_{n} T+r\right)-g_{P}(\tau)\right\| \mathrm{d} \tau \\
& \quad+\int_{t_{-\infty}}^{t_{m}} e^{-\alpha\left(t_{m}-\tau\right)}\left\|P\left(\tau+k_{l} T+r\right) f\left(\tau+k_{l} T+r\right)-g_{P}(\tau)\right\| \mathrm{d} \tau \\
& \quad+\int_{t_{-\infty}}^{t_{m}} e^{-\alpha\left(t_{m}-\tau\right)}\left\|P\left(\tau+k_{l} T+r\right) f\left(\tau+k_{l} T+r\right)-g_{P}(\tau)\right\| \mathrm{d} \tau \\
& \quad+\int_{t_{m}}^{+\infty} e^{\alpha\left(t_{m}-\tau\right)}\left\|Q\left(\tau+k_{n} T+r\right) f\left(\tau+k_{n} T+r\right)-g_{Q}(\tau)\right\| \mathrm{d} \tau \\
& \quad+\int_{t_{m}}^{+\infty} e^{\alpha\left(t_{m}-\tau\right)}\left\|Q\left(\tau+k_{n} T+r\right) f\left(\tau+k_{n} T+r\right)-g_{Q}(\tau)\right\| \mathrm{d} \tau \\
& \quad+\int_{t_{m}}^{+\infty} e^{\alpha\left(t_{m}-\tau\right)}\left\|Q\left(\tau+k_{l} T+r\right) f\left(\tau+k_{l} T+r\right)-g_{Q}(\tau)\right\| \mathrm{d} \tau \\
& \quad+\int_{t_{m}}^{+\infty} e^{\alpha\left(t_{m}-\tau\right)}\left\|Q\left(\tau+k_{l} T+r\right) f\left(\tau+k_{l} T+r\right)-g_{Q}(\tau)\right\| \mathrm{d} \tau
\end{aligned}
$$

Thus,

$$
\begin{aligned}
& \left\|x_{f}\left(t_{m}+k_{n} T+r\right)-x_{f}\left(t_{m}+k_{l} T+r\right)\right\| \\
& \leq A_{m}^{P}+B_{m, n}^{P}+B_{m, k}^{P}+A_{m}^{Q}+B_{m, n}^{Q}+B_{m, k}^{Q} .
\end{aligned}
$$

The uniform boundedness of the sequences of linear functional

$$
\begin{aligned}
& \phi_{m}: B C(\mathbb{R}) \rightarrow \mathbb{R} \\
& h \mapsto \int_{-\infty}^{t_{m}} e^{-\alpha\left(t_{m}-\tau\right)} h(\tau) \mathrm{d} \tau
\end{aligned}
$$

and

$$
\begin{aligned}
& \times \sigma_{m}: B C(\mathbb{R}) \rightarrow \mathbb{R} \\
& h \mapsto \int_{-\infty}^{t_{m}} e^{-\alpha\left(\tau-t_{m}\right)} h(\tau) \mathrm{d} \tau
\end{aligned}
$$

and the fact that Lemma (3.4) applies to

$$
\begin{aligned}
& P\left(k_{n} T+.\right) f\left(k_{n} T+.\right)-g_{P}(.) \\
& \text { and } Q\left(k_{n} T+.\right) f\left(k_{n} T+.\right)-g_{Q}(.)
\end{aligned}
$$

By going to appropriate subsequences, we can assume that the iterated double limits for $\left\{B_{n, m}^{P}\right\}_{n, m \in \mathbb{N}}$ (resp. for $\left\{B_{n, m}^{Q}\right\}_{n, m \in \mathbb{N}}$ ) exist. Since they have to coincide, they have to be zero. By the triangle inequality we find,

$$
\begin{aligned}
& \left\|x_{f}\left(t_{m}+k_{n} T+r\right)-x_{f}\left(t_{m}+k_{l} T+r\right)\right\| \\
& \leq A_{m}^{P}+B_{m, n}^{P}+B_{m, k}^{P}+A_{m}^{Q}+B_{m, n}^{Q}+B_{m, k}^{Q} .
\end{aligned}
$$

Starting with $\lim _{l \rightarrow \infty}$, then $\lim _{m \rightarrow \infty}$, and at last $\lim _{n \rightarrow \infty}$, we obtain

$$
\lim _{m \rightarrow \infty} \lim _{l \rightarrow \infty}\left\langle x_{f}\left(w_{l}+t_{m}\right), x_{m}^{*}\right\rangle=\lim _{n \rightarrow \infty} \lim _{m \rightarrow \infty}\left\langle x_{f}\left(w_{l}+t_{m}\right), x_{m}^{*}\right\rangle,
$$

which concludes the proof.

\section{Acknowledgements}

The authors would like to thank the I. R. D. (Institut de
Recherche pour le Développement, UMI 209) for its hospitality and support.

\section{REFERENCES}

[1] C. Corduneanu, "Almost Periodic Functions," Wiley, New York, 1968.

[2] A. M. Fink, "Almost Periodic Differential Equations," Springer-Verlag, New York, 1974.

[3] C. Zhang, "Almost Periodic Type Functions and Ergodicity," Science Press, Beijing; Kluwer Academic Publishers, Dordrecht, 2003.

[4] E. Ait Dads, “Contribution à l'existence de Solutions Presque Périodiques d'une Équation Fonctionnelle non Linéaire," Thèse d'Etat, Faculté des Sciences Semlalia, Université Cadi Ayyad, Marrakech, 1994.

[5] E. Ait Dads and K. Ezzinbi, "Existence of Positive Pseudo-Almost-Periodic Solutions for Some Nonlinear Infinite Delay Integral Equations Arising in Epedimic Problems," Nonlinear Analysis, Theory, Methods and Applications, Vol. 41, No. 1-2, 2002, pp. 1-13.

[6] E. Ait Dads and K. Ezzinbi, "Pseudo-Almost-Periodic Solutions for Some Delay Differential Equations," Journal of Mathematical Analysis and Applications, Vol. 201, No. 3, 1996, pp. 840-850. doi:10.1006/jmaa.1996.0287

[7] W. F. Eberlein, "Eberlein Weak Almost Periodicity and Differential Equations in Banach Spaes," Ph.D. Thesis, Universitat Essen, Germany, 1992.

[8] W. M. Ruess and W. H. Summers, "Weak Almost Periodicity and the Strongly Ergodic Limit Theorem for Conraction Semigroups," Israel Journal of Mathematics, Vol. 64, 1988, pp. 139-157.

[9] W. M. Ruess and W. H. Summers, "Weak Almost Periodicity and the Strongly Ergodic Limit Theorem for Periodic Evolution Systems," Journal of Functional Analysis, Vol. 94, No. 1, 1990, pp. 177-195. doi:10.1016/0022-1236(90)90033-H

[10] W. M. Ruess and W. H. Summers, "Weak Almost Periodic Semigroups of Operators," Pacific Journal of Mathematics, Vol. 143, 1990, pp. 175-193.

[11] W. M. Ruess and W. H. Summers, "Integration of Asymptotically Almost Periodic Functions and Weak Asymptotic Almost Periodicity," Dissertationes Mathematicae, Vol. 279, 1989.

[12] J. Kreulich, "Weakly Almost-Periodic Solutions of Evolution Equations in Banach Spaces," Differential Integral Equations, Vol. 5, No. 9, 2011, pp. 1005-1027.

[13] J. Kreulich, "Eberlein-Weakly Almost-Periodicity Sand Differential Equations in Banach Spaces," Ph.D. Thesis, University Essen, Germany, 1992.

[14] E. Ait Dads, K. Ezzinbi and S. Fatajou, "Weakly Almost Periodic Solutions for Some Differential Equations in a Banach Space," Nonlinear Studies, Vol. 4, No. 2, 1997, pp. $157-170$

[15] E. Ait Dads, K. Ezzinbi and S. Fatajou, "Weakly Almost Periodic Solutions for the Inhomogeneous Linear Equations and Periodic Processes in a Banach Space," Dy- 
namic Systems and Applications, Vol. 6, 1997, pp. 507516.

[16] E. Ait Dads, K. Ezzinbi and S. Fatajou, "Asymptotic Behaviour of Solutions for Some Differential Equations in Banach Spaces," African Diaspora Journal of Mathematics, Vol. 12, No. 1, 2011, pp. 1-18.

[17] H. Liu James, G. M. N'Guérékata and N. Van Minh, "Topics on Stability and Periodicity in Abstract Differential Equations," Series on Concrete and Applicable Mathematics, Vol. 6, 2008.

[18] K. De Leeuw and I. Glicksberg, "Applications of Almost Periodic Compactifications," Acta Mathematica, Vol. 105, No. 1-2, 1961, pp. 63-97. doi:10.1007/BF02559535
[19] K. De Leeuw and I. Glicksberg, "Almost Periodic Functions on Semigroups," Acta Mathematica, Vol. 105, No. 1-2, 1961, pp. 99-140. doi:10.1007/BF02559536

[20] U. Krengel, "Ergodic Theorems," De Gruyter Studies in Mathematical, 1985. doi:10.1515/9783110844641

[21] W. M. Ruess and W. H. Summers, "Ergodic Theorems for Semigroups of Operators," Proceedings of the American Mathematical Society, Vol. 114, No. 2, 1992, pp. 423-432.

[22] A. Grothendieck, "Critères de Compacité dans les Espaces Fonctionnels Généraux," American Journal of Mathematics, Vol. 74, No. 1, 1952, pp. 168-186. doi: $10.2307 / 2372076$ 